Revista Científica de FAREM-Estelí

Medio ambiente. tecnología y desarrollo humano

Año 10 | Núm. 40 | Octubre-diciembre, 2021

ISSN: 2305-5790

htt ps://rcientificaesteli.unan.edu.ni

DOI: https://doi.org/10.5377/farem.v10i40.13048

\section{Microzonificación sísmica del sector urbano de la ciudad de León, Nicaragua}

\section{Seismic microzonation of the urban sector of the city of León, Nicaragua}

\section{Nadir Castrillo-Osorio}

Instituto de Geología y Geofísica (IGG/UNAN-Managua)

https://orcid.org/0000-0002-4541-9601

edwin.castrillo@igg.unan.edu.ni

\section{RESUMEN}

Los objetivos principales de esta investigación fueron determinar la demanda sísmica así como definir la microzonificación sísmica en el sector urbano de la ciudad de León, este sector tiene alrededor de173,866 Habitantes, este es el número de beneficiados directos con la información producida en esta investigación. En esta investigación utilizamos medición de vibración ambiental (MHVSR), así como medición de ondas superficiales con el método activo MASW. Las funciones de transferencia empíricas(FTE) mostraron que los suelos del área vibran con tres modos, los cuales varían su periodo de vibración entre los valores To $1=0.049$ a $0.160 \mathrm{~s}$, To $2=0.161$ a0.330 s y To3 $=0.331$ a 0.969 s. Además, basado en los resultados de Vs30 y en el RNC-07 los suelos del área pueden clasificarse como suelos firmes o suelo moderadamente blando o como tipo II y III, respectivamente. Los resultados de esta investigación serán utilizados con fines de fortalecer la mitigación de desastres contra terremotos en esta ciudad al ser incluidos en el reglamento nacional de construcción nicaragüense.

\section{ABSTRACT}

The main objectives of this research were to determine the seismic demand as well as to define the seismic microzonation in the urban sector of the city of Leon, this sector has about 173,866 inhabitants, this is the number of direct beneficiaries with the information produced in this research. In this research we used environmental vibration measurement (MHVSR), as well as surface wave measurement with the MASW active method. The empirical transfer functions(ETF) showed that the soils in the area vibrate with three modes, which vary their vibration period between the values To $1=0.049$ to $0.160 \mathrm{~s}$, To $2=0.161$ to $0.330 \mathrm{~s}$ and To $3=0.331$ to $0.969 \mathrm{~s}$. In addition, based on the results of Vs30 and the RNC-07 the soils in the area can be classified as firm soils or moderately soft soil or as type II and III, respectively. The results of this research will be used to strengthen disaster mitigation against earthquakes in this city by being included in the Nicaraguan national construction regulations.
RECIBIDO

$22 / 10 / 2021$

ACEPTADO

$11 / 12 / 2021$

\section{PALABRAS CLAVE}

Microzonificación sísmica; respuesta sísmica; ondas sísmicas superficiales; MASW; UNAN-Managua.

\section{KEYWORDS}

Seismic microzonation; seismic response; surface seismic waves; MASW; UNAN-Managua. 


\section{INTRODUCCIÓN}

La actividad sísmica en Nicaragua se genera de tres fuentes principales las cuales son la zona de subducción, la cadena volcánica y el fallamiento local. Siendo la zona de subducción nicaragüense y el fallamiento local las fuentes sísmicas que mayormente afectan a las ciudades del Pacifico nicaragüense. La actividad sísmica en la ciudad de León, aunque no es frecuente, en la memoria histórica se tiene registros de daños causados por sismos de considerable magnitud, los cuales generaron destrucción, los eventos sísmicos más recientes que generaron daños en esta ciudad son los de 1919 y 1951. En el sismo de 1919 se produjeron daños entre los que resaltan el colapso de una de las torres de la iglesia de Zaragoza, en tanto el terremoto de 1951 también genero daños considerables siendo uno de los más recordados el colapso parcial de la iglesia del Laborío (MOVIMONDO, 2004).

Es un acuerdo unánime en la comunidad científica en el campo de la ingeniería sísmica-sismológica que uno de los factores que más contribuyen a elevar el potencial destructivo de las ondas sísmicas son las condiciones locales de los suelos en un sitio (Castrillo-Osorio, 2021; Bonnefoy-Claudet et al., 2006; Kagawa, 1996). Por lo cual es fundamental en zonas sísmicas estudiar y caracterizar las propiedades dinámicas de los suelos y como estos responderán ante un terremoto, de tal manera que podamos anticipar el efecto del terremoto en las viviendas y edificaciones.

El inicio del estudio de los microtremores fue desarrollado por Nogoshi \& Igarashi (1971) basándose en el estudio por Kanai \& Tanaka (1961). Esta es una de las metodologías más ampliamente utilizadas para determinar la frecuencia fundamental de vibración de un depósito de suelo, la cual es una de las propiedades dinámicas más importantes en el campo de la ingeniería sísmica. Los microtremores son movimientos generados por los procesos naturales de la tierra, por ejemplo, el viento, las olas en el océano, los cambios atmosféricos así como por fuentes artificiales antropogénicas como pueden ser maquinaria en las industrias, trafico, entre otros. Los microtremores son movimientos débiles del suelo cuyas amplitudes varían entre 10-4 a 10-2 $\mathrm{mm}$.

En Nicaragua, a partir del año 2000, estudios basados en microtremores así como de velocidades de ondas de corte (Vs) han sido desarrollados por algunos autores, el Instituto Nicaragüense de Estudios Territoriales (INETER, 2000) realizo el estudio de microzonificación sísmica de la ciudad de Managua el cual estuvo basado en la técnica de MHVSR o de Nakamura, Parrales \& Ulriksen (2006), realizaron estudio de las propiedades dinámicas en sitios seleccionados de los suelos de la ciudad de Managua, Romero (2005) realizo el estudio de efecto de sitio basado en microtremores en la ciudad de Masaya, Obando et al (2011) estudiaron las funciones de transferencias a partir de 
un arreglo móvil, Hernández \& Lermo (2009) realizaron la reevaluación de los periodos del efecto de sitio y propuesta de clasificación de terreno con fines de diseño sísmico para la ciudad de Managua, Martínez (2011) estudio el efecto de la geología superficial en los movimientos sísmicos en Managua, Castrillo-Osorio et al (2014) realizaron el estudio para determinar el efecto de sitio en el antiguo centro histórico de la ciudad de Managua con microtremores de periodo largo.

En la ciudad de León, a pesar que se han realizado algunos estudios para determinar ciertas características dinámicas de los suelos (Lacayo, 2016), asi como para definir parámetros sísmicos de movimiento del suelo como el estudio realizado por Shah et al (1975), este estudio constituye el primero en su tipo, esto dado que, hasta el momento de la presentación de los resultados de este proyecto de investigación las características dinámicas rslsnde los suelos de la ciudad de León no habían sido estudiadas de acuerdo a las guías actuales que dan las últimas recomendaciones sobre la adecuada realización de estos estudios, por lo cual, previo a este estudio era imposible aun caracterizar con certeza el comportamiento de los suelos de León ante terremotos futuros, es por ello que este estudio tiene un enorme valor para fortalecer la resiliencia ante terremotos de la ciudad.

La medición de propiedades dinámicas en este estudio se realizó utilizando microtremores, cuyos datos al ser analizados permiten determinar el periodo fundamental de vibración de un sitio (Nakamura, 1989). En su estudio Nakamura (1989) propuso el método de razón espectral en el cual el periodo fundamental de vibración es determinado mediante el cociente de los espectros de Fourier de las componentes horizontales y vertical para un mismo sitio.

Adicionalmente, en este estudio fue necesario determinar perfiles de velocidades de ondas del suelo hasta $30 \mathrm{~m}$ de profundidad, la manera más convencional de realizar esto es utilizando pruebas de borehole, sin embargo, esto previamente a la prueba involucra realizar perforaciones las cuales son bastante costosas y de extensa duración en comparación a un levantamiento sísmico, lo cual hace que este método no sea el más adecuado para trabajos de microzonificación (Tuladhar et al., 2004).

Otra posibilidad es utilizar registros de terremotos que estén disponibles en estaciones sísmicas de la red nacional, sin embargo, en Nicaragua existen muy pocos registros de terremotos como para utilizar esta metodología. Por lo cual en este estudio optamos por el método multicanal de ondas superficiales (MASW)(Park et al., 1999), el cual consiste en generar de forma artificial en la superficie del suelo ondas sísmicas superficiales a partir de las cuales podemos obtener la cantidad de registros necesarios para determinar el perfil de velocidad de un sitio dado. 
Las características dinámicas obtenidas nos permitieron determinar una microzonificación sísmica, primeramente, basada en periodos fundamentales de vibración, así como una microzonificación basada en la distribución espacial de ondas $\mathrm{S}$, y estimar la demanda sísmica en la zona urbana de la ciudad de León. Adicionalmente, otro de los aspectos importantes de esta investigación, es el de mitigar desastres que pueden ser causados por terremotos futuros en la ciudad de León a través de la producción de información que permita a los tomadores de decisiones planear el crecimiento urbano de la ciudad, así como producir la actualización del Reglamento Nicaragüense de construcción.

Los objetivos principales de esta investigación fueron determinar la demanda sísmica, así como definir la microzonificación sísmica en el sector urbano de la ciudad de León, este sector tiene alrededor de 173,866 Habitantes de acuerdo al último censo del Instituto Nacional de Estadísticas (INIDE, 2020), este es el número de beneficiados directos con la información producida en esta investigación

\section{MATERIALES Y MÉTODOS}

\section{Área de estudio}

El universo de esta investigación correspondió al sector urbano de la ciudad de León (Figura 1), la muestra corresponde a los registros sísmicos adquiridos específicamente, 40 registros de microtremores y 11 registros de ondas sísmicas superficiales, el tipo de investigación desarrollada en este estudio corresponde a una investigación cuantitativa.

Geológicamente la litología de los suelos superficiales de León corresponde a suelos de origen volcánico (Hodgson, 2000). A escala regional, León está asentada dentro de una estructura geológica en forma de franja que se extiende casi paralela a la costa Pacífica de Nicaragua denominada depresión de Nicaragua. Esta estructura ubicada en la región del Pacifico tiene una dirección aproximada de N45W y un ancho aproximado de $75 \mathrm{~km}$. El Instituto Nicaragüense de Estudios Territoriales determino que dos fallas normales limitan la depresión por lo cual ha sido clasificada como Graben (INETER, 2004). 
Figura 1. El polígono muestra el sector urbano de la ciudad de León. Los cuadrados representan los sitios donde se adquirieron datos de microtremores, mientras que los pentágonos marcan los sitios donde se realizaron mediciones de ondas sísmicas superficiales

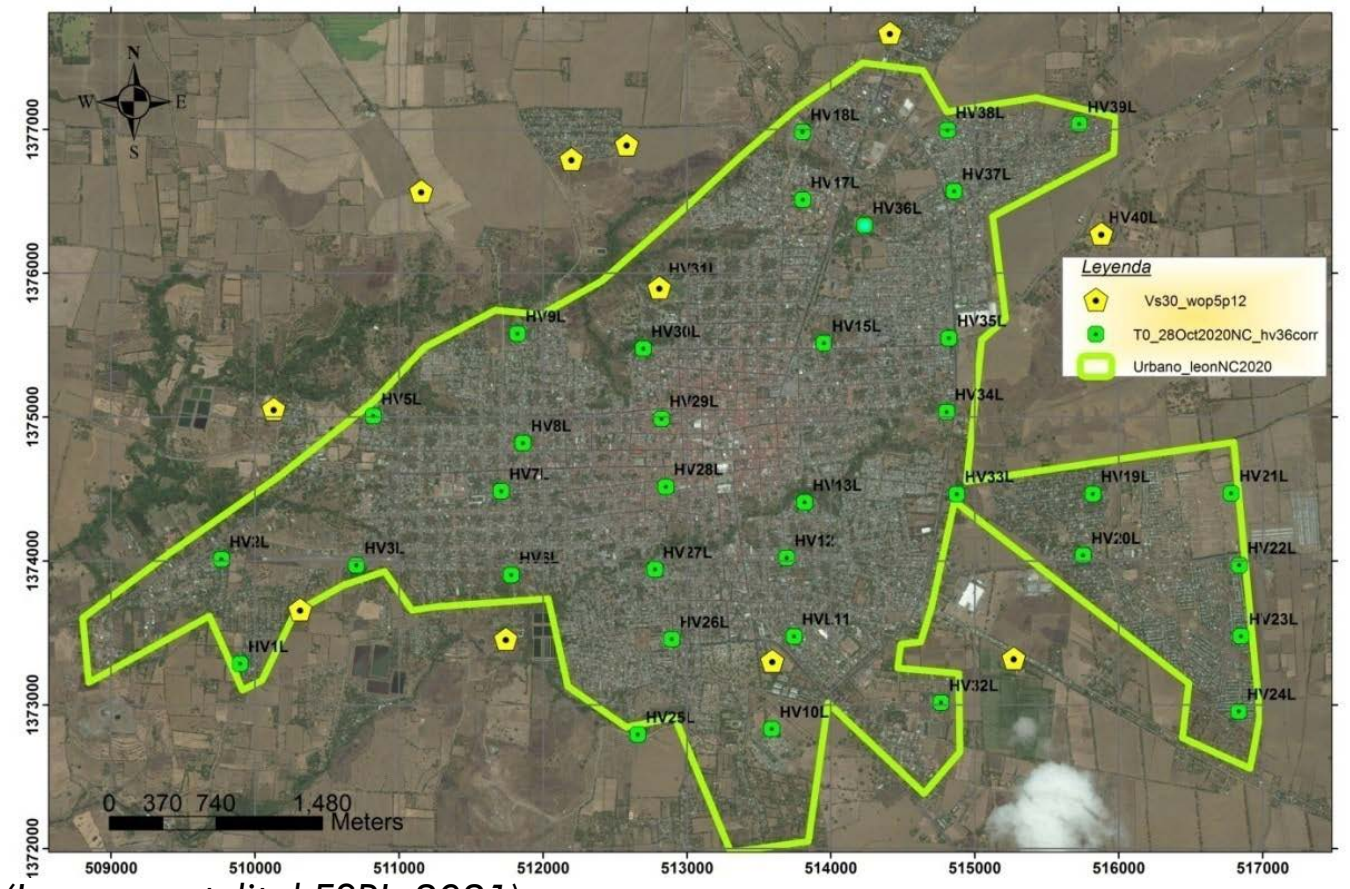

(Imagen satelital ESRI, 2021).

Se utilizaron dos métodos sísmicos de campo para obtener los datos en esta investigación, los cuales fueron, el método de microtremores o de cocientes espectrales (MHVSR). El método de microtromeres se basa en el registro de vibraciones del suelo muy débiles en el orden de micrómetros, las principales fuentes de estas vibraciones son la naturaleza, así como las actividades humanas. Este método tiene como resultado la determinación del modo fundamental de vibración de los suelos, este dato es muy importante pues permite diseñar las construcciones futuras para que su periodo natural de vibración sea diferente al del suelo. Existen casos históricos donde desafortunadamente el desconocimiento de este parámetro llevo al colapso de múltiples edificios de apartamentos durante el terremoto de Michoacán en México en 1985, esto ocurrió producto de un fenómeno llamado de resonancia donde la coincidencia del periodo de vibración del suelo con el del edificio amplifica exponencialmente la vibración generada por las ondas sísmicas del terremoto en la edificación.

Además se utilizó el método multicanal de análisis de ondas superficiales (MASW). Adicionalmente se utilizaron métodos numéricos basados en la teoría de multireflexión de ondas sísmicas para el cálculo de la respuesta sísmica de sitio basado en el comportamiento dinámico de los suelos ante un terremoto futuro. A partir de la información de respuesta sísmica, se logró estimar la demanda sísmica, con cuya información los diseñadores de obras 
civiles pueden proyectar las edificaciones de acuerdo al contexto sísmico de la zona.

Esta investigación se dividió en las fases descritas a continuación:

Trabajo de Gabinete: Primeramente, fue necesario recolectar cualquier información de estudios anteriores realizados en la ciudad ya sea en la temática de la investigación o de estudios base como cartografía, topografía, geología y geotecnia.

Trabajo de Campo: Las mediciones en campo se realizaron siguiendo dos métodos, en primer lugar, se realizaron mediciones de vibración ambiental mediante el método HVSR o técnica de (Nakamura, 1989). La adquisición de registros de microtremores se realizó en 40 sitios ubicados en el sector urbano de la ciudad de León (Figura 1), para esto utilizamos el acelerógrafo McSeis MT-Neo acoplado con sismómetros de periodo largo.

Adicionalmente se realizó adquisición de registros sísmicos mediante el método de ondas superficiales o MASW (Park et al., 1999). Este método permite la caracterización desde la superficie del suelo hasta determinada profundidad de la propiedad dinámica del suelo conocida como velocidad de ondas de corte del mismo. En este estudio se logró alcanzar profundidades de hasta $30 \mathrm{~m}$, determinar este parámetro es fundamental para posteriormente calcular la respuesta sísmica de sitio dado que de la velocidad de ondas de corte de los estratos depende como las ondas sísmicas serán amplificadas o atenuadas.

Figura 2. A la izquierda se muestra el sistema de adquisición para registro de vibración ambiental. A la derecha se observa el arreglo lineal desplegado para la adquisición de ondas sísmicas superficiales, estas son generadas con una fuente artificial usando un mazo de $20 \mathrm{lb}$ como generador.

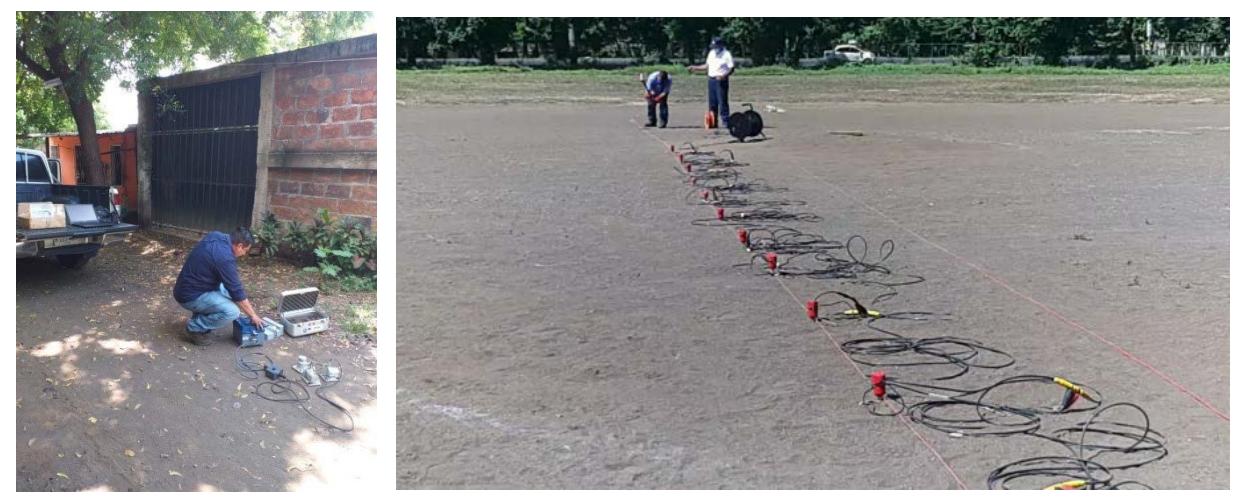

La adquisición de registros de ondas sísmicas superficiales se realizó en 11 sitios ubicados en el área de estudio (Figura 1), para esto utilizamos un sismómetro con 24 canales conectado a un disparador piezoeléctrico el cual le indicaba al sistema el arribo de la primera onda sísmica para iniciar el registro de las mismas. 


\section{Procesamiento de datos:}

El procesamiento de los registros de microtremores se realizó siguiendo los criterios establecidos por (Nakamura, 1989) según el cual de modo general se realiza la norma de las componentes generales sobre la componente vertical, razón por la cual este método también se conoce como razón espectral de $\mathrm{H}$ sobre $\mathrm{V}$. A partir de este procedimiento obtuvimos los valores del periodo fundamental de vibración para los suelos de cada uno de los sitios donde se realizaron mediciones.

Durante el análisis de los resultados de microtremores, primeramente, se obtuvieron datos de familias de curvas mediante la herramienta de procesamiento para determinar el cociente espectral (Wathelet et al., 2020). Luego estos datos se introdujeron en un software de interpolación (Arcmap) y este nos produjo la agrupación espacial de zonas con periodos fundamentales de vibración del suelo que eran similares en el área de estudio.

Posteriormente los registros de ondas sísmicas superficiales fueron procesados siguiendo el método de transformación desarrollado por Park et al (1999) mediante el cual los registros de la configuración de campo en tiempo espacio $(x-t)$ se transforman a fase-frecuencia (C-f). A partir de la transformación se obtuvo la relación frecuencia-velocidad o espectro de velocidad, y luego mediante inversión se determinó el modelo unidimensional de ondas sísmicas de corte (Vs) en cada uno de los sitios donde se adquirieron datos de ondas sísmicas superficiales.

\section{Simulaciones numéricas:}

El método numérico utilizado fue el de análisis lineal equivalente, mediante el cual fue posible calcular como las características del emplazamiento del suelo influenciaban la respuesta sísmica de sitio en el área de estudio. El método, se basa en un ajuste iterativo entre la deformación cortante efectiva obtenida de los cálculos y las curvas de degradación tomadas de la bibliografía (Arslan \& Siyahi, 2006; Schnabel \& Seed, 1972). Para el cálculo de aceleraciones espectrales se hizo uso de los modelos Vs estimados con el método MASW.

Las curvas dinámicas (módulo de reducción y amortiguamiento) para modelar la respuesta de sitio se seleccionaron de la literatura en este caso para suelo granulares o suelos no cohesivos. En esta investigación se utilizaron las curvas de Seed e Idriss (1970). La selección de estas curvas se justifica por las características geológicas de los suelos de la ciudad de León.

La selección de los registros de terremotos de entrada para el análisis se hizo utilizando el método de espectro condicional promedio (Baker \& Lee, 2018). El espectro condicional se ajustó para fijar el espectro objetivo equivalente al espectro de amenaza uniforme para 500 años calculado para la ciudad de León. Cada registro, se asumió como el registro de entrada en la parte inferior 
del modelo de suelo a analizar. Cada uno de los registros fue debidamente seleccionado para que cumpliera con las amplitudes en roca de acuerdo al espectro determinado mediante el análisis de amenaza sísmica.

En este estudio utilizamos la teoría de propagación de ondas en una dimensión, así como el modelamiento lineal equivalente para la evaluación aproximada de la respuesta de sitio no lineal, el cual ha probado dar resultados aproximados a la respuesta no lineal de los suelos (Arslan \& Siyahi, 2006; Schnabel et al., 1972).

Tabla 1. Características de los terremotos utilizados para el análisis de respuesta sísmica de sitio.

\begin{tabular}{|c|c|c|c|}
\hline Nombre del terremoto & Año & Nombre de la estación & Magnitud \\
\hline "Mateare" & 2014 & --- & 6.2 \\
\hline "Managua" & 1972 & ESSO & 6.2 \\
\hline "Imperial Valley-06" & 1979 & "Cerro Prieto" & 6.53 \\
\hline "Livermore-01" & 1980 & "APEEL 3E Hayward CSUH" & 5.8 \\
\hline "Livermore-01" & 1980 & "Del Valle Dam (Toe)" & 5.8 \\
\hline "Livermore-02" & 1980 & "APEEL 3E Hayward CSUH" & 5.42 \\
\hline "Livermore-02" & 1980 & "Del Valle Dam (Toe)" & 5.42 \\
\hline "Mammoth Lakes-03" & 1980 & "Long Valley Dam (Downst)" & 5.91 \\
\hline "Mammoth Lakes-03" & 1980 & "Long Valley Dam (L Abut)" & 5.91 \\
\hline "Mammoth Lakes-06" & 1980 & "Benton" & 5.94 \\
\hline "Mammoth Lakes-06" & 1980 & "Bishop - Paradise Lodge" & 5.94 \\
\hline "Westmorland" & 1981 & "Superstition Mtn Camera" & 5.9 \\
\hline "Morgan Hill" & 1984 & "Gilroy - Gavilan Coll." & 6.19 \\
\hline "Chalfant Valley-01" & 1986 & "Benton" & 5.77 \\
\hline "Chalfant Valley-01" & 1986 & "Bishop - Paradise Lodge" & 5.77 \\
\hline "Chalfant Valley-01" & 1986 & "Lake Crowley - Shehorn Res." & 5.77 \\
\hline "Chalfant Valley-02" & 1986 & "Benton" & 6.19 \\
\hline "Chalfant Valley-02" & 1986 & "Convict Creek" & 6.19 \\
\hline "Chalfant Valley-03" & 1986 & "Bishop - Paradise Lodge" & 5.65 \\
\hline "Landers" & 1992 & "Silent Valley - Poppet Flat" & 7.28 \\
\hline "Landers" & 1992 & "Twentynine Palms" & 7.28 \\
\hline "Big Bear-01" & 1992 & $\begin{array}{c}\text { "Rancho Cucamonga - Law and Justince } \\
\text { Center FF_Foothill and Haven" }\end{array}$ & 6.46 \\
\hline "Big Bear-01" & 1992 & "Riverside Airport" & 6.46 \\
\hline "Big Bear-01" & 1992 & "Silent Valley - Poppet Flat" & 6.46 \\
\hline "Big Bear-01" & 1992 & "Wrightwood - Nielson Ranch" & 6.46 \\
\hline "Kobe_Japan" & 1995 & "Chihaya" & 6.9 \\
\hline "Kocaeli_Turkey" & 1999 & "Mecidiyekoy" & 7.51 \\
\hline "Duzce_Turkey" & 1999 & "Sakarya" & 7.14 \\
\hline
\end{tabular}




\begin{tabular}{|c|c|c|c|}
\hline "Caldiran_Turkey" & 1976 & "Maku" & 7.21 \\
\hline "Hector Mine" & 1999 & "Fun Valley" & 7.13 \\
\hline "Hector Mine" & 1999 & "Twentynine Palms" & 7.13 \\
\hline "Big Bear-01" & 1992 & "Highland Fire Station" & 6.46 \\
\hline "Big Bear-01" & 1992 & "Fun Valley" & 6.46 \\
\hline
\end{tabular}

(Ancheta et al., 2014)336 (mostly.

En la tabla 1 se muestran algunos datos de los terremotos de entrada que se utilizaron para el análisis, cuyas magnitudes varían entre 5.0 a 6.2. Los parámetros sismológicos utilizados son similares a los de los terremotos locales que ocurren en la ciudad de León. Quince de estos registros se tomaron de la base de datos del Pacific Earthquake Engineering Research Center (Ancheta et al., 2014)336 (mostly. Además, se incluyó en el análisis el registro del terremoto de Managua de 1972.

\section{Clasificación sísmica de sitio:}

De acuerdo al Reglamento Nacional de la Construcción vigente en Nicaragua (MTI, 2007) y la National Earthquake Hazard Reduction Program (FEMA, 2003), es posible clasificar los suelos basados en Vs de acuerdo a la siguiente ecuación:

$$
V S=\frac{\sum_{n-1}^{N} h_{n}}{\sum_{n-1}^{N} \frac{h_{n}}{V_{n}}}
$$

Ec. 1

Donde:

$\mathrm{hn}=$ espesor del enésimo estrato.

$V_{n}=$ velocidad de ondas de corte del enésimo estrato.

$\mathrm{N}=$ número de estratos.

Los resultados de los perfiles de velocidades de ondas $\mathrm{S}$ obtenidos mediante la técnica de MASW se muestran a continuación:

\section{Espectro de diseño:}

Se calcularon espectros de diseño para sitios seleccionados del área en estudio siguiendo la formulación de RNC-07 (MTI, 2007):

$$
a=\left\{\begin{array}{c}
S\left[a_{0}+\left(d-a_{0}\right) \frac{T}{T_{a}}\right] ; \text { if } T<T_{a} \\
S d ; \quad \text { if } T<T_{a} \\
S d\left(\frac{T_{b}}{T}\right) ; \text { if } T_{b} \leq T \leq T_{c} \\
S d\left(\frac{T_{b}}{T_{c}}\right)\left(\frac{T_{c}}{T}\right)^{2} ; \quad \text { if } T>T_{c}
\end{array}\right.
$$


Donde:

a es la ordenada del espectro de diseño en términos de aceleración.

$a_{0}$ es la aceleración tomada del mapa de isoaceleraciones cuando $\mathrm{T}=0$.

$d$ es un coeficiente con valores

$\mathrm{S}$ es un coeficiente de amplificación cuyo valor depende del tipo de suelo y esta especificado en RNC-07.

$T$ es el periodo estructural en segundos.

$T_{a}$ Es el valor del periodo estructural que limita la parte ascendente del espectro de diseño con, en segundos.

$T_{b}$ Es el valor del periodo estructural que limita donde inicia el decremento de las ordenadas del espectro de diseño luego de la meseta con en segundos, y que se extiende hasta.

$T_{c}$ Es el valor del periodo estructural que limita donde inicia el segundo cambio de pendiente en el decremento del espectro de diseño con en segundos, y que se extiende hasta infinito.

\section{ANÁLISIS Y DISCUSIÓN DE RESULTADOS}

\section{Distribución de períodos de vibración del suelo en el área de estudio}

En el presente estudio se ha evidenciado que, en general, la información determinada con nuestros resultados coincide con los estudios realizados antes en la ciudad, por ejemplo Lacayo (2016) encontraron en la zona céntrica de la ciudad y sectores aledaños, periodos fundamentales de vibración que variaban en el rango de valores desde 0.3 hasta $0.9 \mathrm{~s}$, podemos ver de la Figura 3 que la franja color café contiene este mismo rango de valores reportados en el estudio mencionado. Sin embargo la extensión de nuestra área de estudio es mayor que la estudiada por Lacayo (2016), además, el nivel de detalle de las mediciones realizadas en este estudio aporta mayor claridad a posibles zonas de transición así como de microzonas que los estudios anteriores no habían develado (Figura 3). Por lo anterior en este estudio, hemos definido microzonas "nuevas" respecto al estudio mencionado.

El estudio de Lacayo (2016) clasifico la zona central del presente estudio como suelos muy densos a suelos moderadamente blandos solamente basándose en resultados de microtremores, sin embargo en el presente estudio, además de los microtremores, se determina una microzonificación basada en velocidades de ondas de corte, este dato adicional valida la clasificación por microtremores, además se dejan definidas espacialmente las zonas donde existe un tipo de suelo firme y donde existe el moderadamente blando (ver Figura 5). 
De acuerdo a los resultados, los suelos del sitio pueden clasificarse como suelos firmes a suelos moderadamente blandos. Lo antes mencionado tiene implicaciones muy importantes, porque por ejemplo si un diseñador estructural utiliza los datos basados solamente en microtremores y con poca resolución de los estudios anteriores, puede diseñar su edificio con la demanda sísmica más baja asumiendo suelo firme en un sitio, en cambio al tener adicionalmente una microzonificación por velocidades de ondas de corte puede conocer si su sitio esta efectivamente en suelo firme o moderadamente blando y diseñar de acuerdo a la demanda sísmica que requerirán los futuros eventos sísmicos a su edificación según contexto sísmico.

Se puede observar (Figura 3) que el área ha quedado dividida en tres microzonas basándonos en periodos fundamentales de vibración:

\section{Figura 3. Mapa de microzonificación sísmica de la ciudad de León basada en periodos fundamentales de vibración (Imagen satelital ESRI, 2021).}

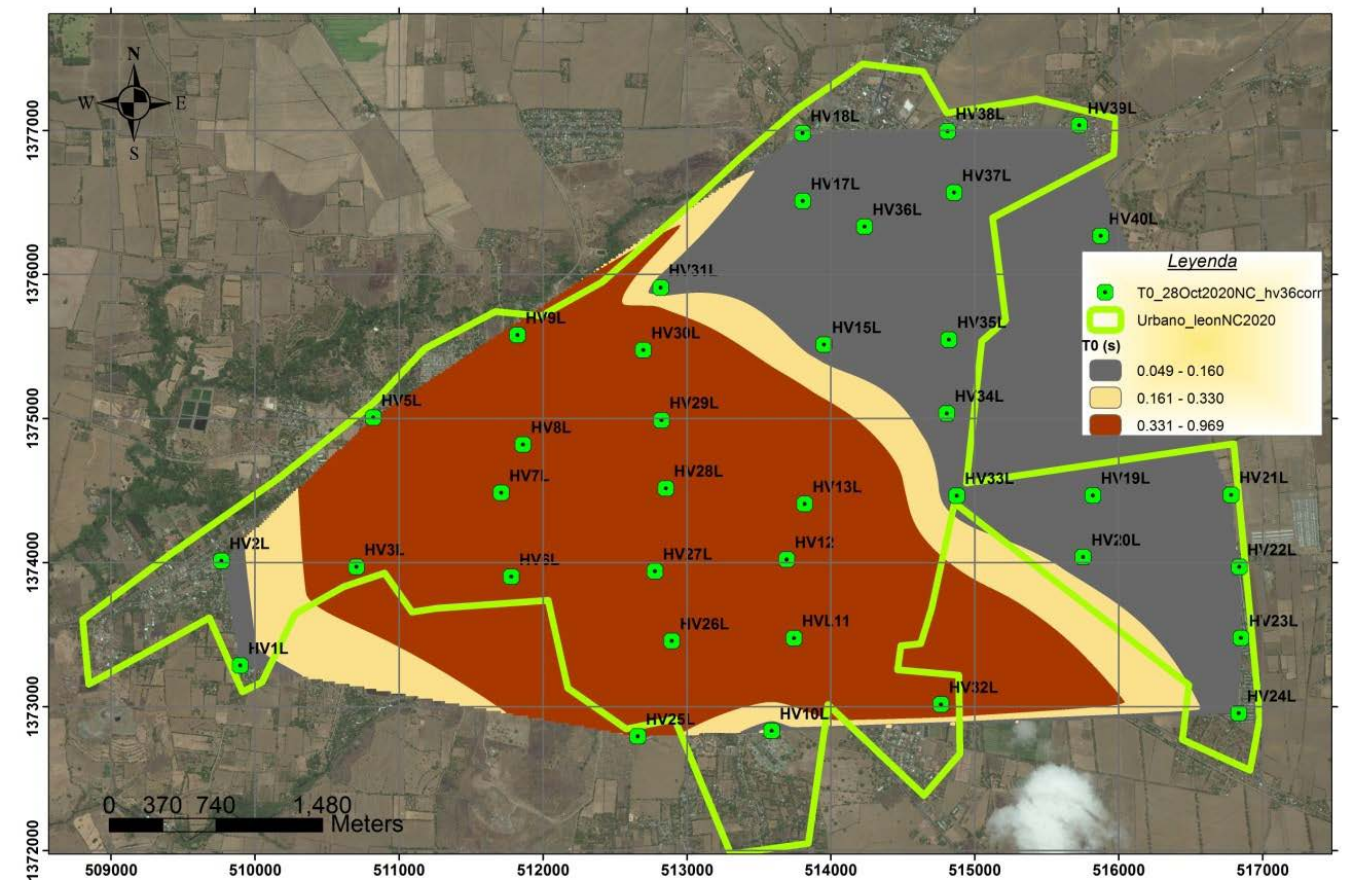

Microzona 1: Presenta periodos de vibración entre 0.049 a $0.160 \mathrm{~s}$, en esta área las edificaciones muy rígidas, así como edificaciones de 1 piso podrían coincidir con la manera de vibrar del suelo. Por lo que se sugiere especial cuidado al edificar este tipo de obras.

Microzona 2: Presenta periodos de vibración entre 0.161 a 0.330 s, en esta área las edificaciones como viviendas de 2 a 3 piso podrían coincidir con la manera de vibrar del suelo. Por lo que se sugiere especial cuidado al edificar este tipo de obras. 
Microzona 3: Presenta peri odos de vibración entre 0.331 a 0.969 s, en esta área las edificaciones como viviendas de 4 a 9 pisos podrían coincidir con la manera de vibrar del suelo. Por lo que se sugiere especial cuidado al edificar este tipo de obras.

\section{Velocidades de ondas S}

En la clasificación sísmica de sitio es importante tomar en cuenta varios parámetros, y uno de los más importantes es la velocidad de ondas de corte $(\mathrm{Vs})$. Este parámetro define de manera confiable las características dinámicas de los materiales (Alfaro et al., 1999). En este estudio se definió la clasificación de zonas de acuerdo a las velocidades de ondas de corte encontradas tomando en cuenta la propuesta de clasificación del Reglamento Nacional de Construcción de Nicaragua del 2007 (MTI, 2007) y la Norma NEHRP (FEMA, 2003). Estas normas recomiendan que el perfil de ondas de corte se debe obtener a una profundidad de 30 metros, lo cual permite obtener una velocidad promedio (Vs30) que caracteriza de manera confiable el sitio, pudiéndose estimar basado en esta información la respuesta sísmica de este, es decir el comportamiento de un sitio ante un terremoto.

Hasta el momento de esta investigación, ningún trabajo ha sido publicado para determinar el parámetro de ondas de corte con la metodología aquí propuesta, por lo cual es una información inédita la que se produjo.

Al realizar un análisis de los resultados de las velocidades de ondas (ver Figura 4), se puede observar que en el sitio del perfil Vs $1 \mathrm{~L}$, los primeros estratos de este suelo presentan valores de velocidad bajos de hasta 200 $\mathrm{m} / \mathrm{s}$. Luego se presenta un contraste alrededor de los $2 \mathrm{~m}$ de profundidad de $380 \mathrm{~m} / \mathrm{s}$. La velocidad promedio hasta los $30 \mathrm{~m}$ de profundidad (Vs30) en este sitio es de $409.7 \mathrm{~m} / \mathrm{s}$ Este suelo puede clasificarse como suelo firme o tipo II.

En el sitio del perfil Vs2L, los primeros estratos de este suelo presentan valores de velocidad medios de hasta $240 \mathrm{~m} / \mathrm{s}$. Luego se presenta un contraste alrededor de los $2 \mathrm{~m}$ de profundidad de $820 \mathrm{~m} / \mathrm{s}$. La velocidad promedio (Vs30) en este sitio es de $545.2 \mathrm{~m} / \mathrm{s}$. Este suelo puede clasificarse como suelo firme o tipo II.

En el sitio del perfil Vs3L, los primeros estratos de este suelo presentan valores de velocidad medios de hasta $240 \mathrm{~m} / \mathrm{s}$. Luego se presenta un contraste alrededor de los $2 \mathrm{~m}$ de profundidad de $820 \mathrm{~m} / \mathrm{s}$. La velocidad promedio (Vs30) en este sitio es de $401.7 \mathrm{~m} / \mathrm{s}$. Este suelo puede clasificarse como suelo firme o tipo II.

En el sitio del perfil $\mathrm{Vs}_{\mathrm{s}} 42 \mathrm{~L}$, los primeros estratos de este suelo presentan valores de velocidad altas de hasta $530 \mathrm{~m} / \mathrm{s}$. Luego se presenta un contraste alrededor de los $3 \mathrm{~m}$ de profundidad de $920 \mathrm{~m} / \mathrm{s}$. La velocidad promedio

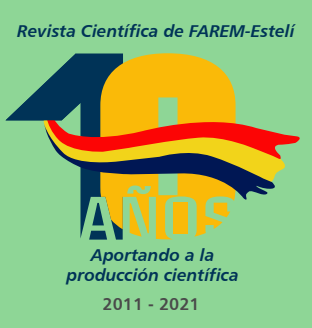


(Vs30) en este sitio es de $924.8 \mathrm{~m} / \mathrm{s}$. Este suelo puede clasificarse como afloramiento rocoso o tipo I.

En el sitio del perfil Vs6L, los primeros estratos de este suelo presentan valores de velocidad medias de hasta $255 \mathrm{~m} / \mathrm{s}$. Luego se presenta un incremento de velocidad en la capa situada alrededor de los $7.20 \mathrm{~m}$ de profundidad de 475 $\mathrm{m} / \mathrm{s}$. La velocidad promedio (Vs30) en este sitio es de $325.6 \mathrm{~m} / \mathrm{s}$. Este suelo puede clasificarse como suelo firme o tipo II.

En el sitio del perfil Vs7L, los primeros estratos de este suelo presentan valores de velocidad medias de hasta $300 \mathrm{~m} / \mathrm{s}$. Luego se presenta una capa con un incremento de velocidad considerable, esta capa está situada alrededor de los $6.25 \mathrm{~m}$ de profundidad y tiene una velocidad $\mathrm{Vs}$ de $510 \mathrm{~m} / \mathrm{s}$. La velocidad promedio (Vs30) en este sitio es de $360.0 \mathrm{~m} / \mathrm{s}$. Este suelo puede clasificarse como suelo firme o tipo II.

En el sitio del perfil Vs8L, los primeros estratos de este suelo presentan valores de velocidad medias de hasta $300 \mathrm{~m} / \mathrm{s}$. Luego se presenta una capa con un incremento de velocidad considerable, esta capa está situada alrededor de los $10.0 \mathrm{~m}$ de profundidad y tiene una velocidad $\mathrm{Vs}$ de $515 \mathrm{~m} / \mathrm{s}$. La velocidad promedio (Vs30) en este sitio es de $354.5 \mathrm{~m} / \mathrm{s}$. Este suelo puede clasificarse como suelo firme o tipo II.

En el sitio del perfil Vs9L, los primeros estratos de este suelo presentan valores de velocidad muy bajas de hasta $30 \mathrm{~m} / \mathrm{s}$. Luego se presenta una capa con un incremento de velocidad considerable, esta capa está situada alrededor de los $2.25 \mathrm{~m}$ de profundidad y tiene una velocidad Vs de $450 \mathrm{~m} / \mathrm{s}$. La velocidad promedio (Vs30) en este sitio es de $354.5 \mathrm{~m} / \mathrm{s}$. Este suelo puede clasificarse como suelo firme o tipo II.

En el sitio del perfil Vs10L, los primeros estratos de este suelo presentan valores de velocidad muy bajas de hasta $70 \mathrm{~m} / \mathrm{s}$. Luego se presenta una capa con un incremento de velocidad, esta capa está situada alrededor de los $9.50 \mathrm{~m}$ de profundidad y tiene una velocidad $\mathrm{Vs}$ de $400 \mathrm{~m} / \mathrm{s}$. La velocidad promedio (Vs30) en este sitio es de $342.65 \mathrm{~m} / \mathrm{s}$. Este suelo puede clasificarse como suelo firme o tipo II.

En el sitio del perfil $V_{s} 11 \mathrm{~L}$, los primeros estratos de este suelo presentan valores de velocidad muy bajas de hasta $100 \mathrm{~m} / \mathrm{s}$. Luego se presenta una capa con un incremento de velocidad, esta capa está situada alrededor de los $2.0 \mathrm{~m}$ de profundidad y tiene una velocidad Vs de $382 \mathrm{~m} / \mathrm{s}$. La velocidad promedio (Vs30) en este sitio es de $633.0 \mathrm{~m} / \mathrm{s}$. Este suelo puede clasificarse como suelo firme o tipo II.

En el sitio del perfil $V_{s} 13 L$, los primeros estratos de este suelo presentan valores de velocidad bajas de hasta $110 \mathrm{~m} / \mathrm{s}$. Luego se presenta una capa con un ligero incremento de velocidad, esta capa está situada alrededor de

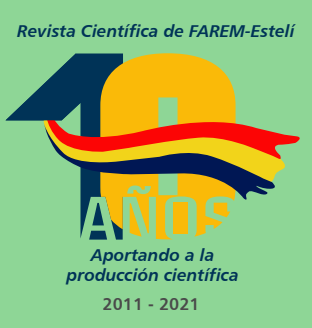


$1.0 \mathrm{~m}$ de profundidad y tiene una velocidad Vs de $262 \mathrm{~m} / \mathrm{s}$. La velocidad promedio (Vs30) en este sitio es de $347.3 \mathrm{~m} / \mathrm{s}$. Este suelo puede clasificarse como suelo moderadamente blando o tipo III.

Utilizando los modelos unidimensionales de velocidades de ondas de corte (ver figura 4) calculados mediante las curvas de dispersión obtenidas con MASW y del análisis anterior, se procedió a realizar una clasificación por tipo de suelo para el área de estudio tomando en cuenta la propuesta de clasificación del NEHRP (FEMA, 2003) así como el RNC-07. En la tabla 2 se observan los resultados usando Vs30 como parámetro para la clasificación sísmica de sitio. Los resultados de todos los perfiles de velocidades de ondas $\mathrm{S}$ hasta 30 metros de profundidad, muestran que el área de estudio se puede definir según las propiedades dinámicas de los suelos como suelo II y III basados en el RNC-07 (MTI, 2007) y como tipo C a D de acuerdo al NEHRP (FEMA, 2003) (Figura 5).

Tabla 2. Clasificación de sitio basada en los criterios.

\begin{tabular}{|c|c|c|c|}
\hline Sitio & Vs30 (m/s) & RNC-07 & NEHRP \\
\hline Vs1L & 409.70 & $\begin{array}{l}\text { Suelo moderadamente } \\
\text { blando (tipo III) }\end{array}$ & $\begin{array}{l}\text { Suelo muy denso y } \\
\text { roca blanda (tipo C) }\end{array}$ \\
\hline Vs2L & 545.22 & $\begin{array}{l}\text { Suelo moderadamente } \\
\text { blando (tipo III) }\end{array}$ & $\begin{array}{l}\text { Suelo muy denso y } \\
\text { roca blanda (tipo C) }\end{array}$ \\
\hline Vs3L & 401.70 & Suelo firme (tipo II) & $\begin{array}{l}\text { Suelo muy denso y } \\
\text { roca blanda (tipo C) }\end{array}$ \\
\hline Vs4L & 924.79 & Afloramiento rocoso (tipo I) & Roca (tipo B) \\
\hline Vs6L & 325.62 & Suelo firme (tipo II) & Suelo firme (tipo D) \\
\hline Vs7L & 360.01 & Suelo firme (tipo II) & $\begin{array}{l}\text { Suelo muy denso y } \\
\text { roca blanda (tipo C) }\end{array}$ \\
\hline Vs8L & 354.52 & $\begin{array}{l}\text { Suelo moderadamente } \\
\text { blando (tipo III) }\end{array}$ & Suelo firme (tipo D) \\
\hline Vs9L & 524.09 & $\begin{array}{l}\text { Suelo moderadamente } \\
\text { blando (tipo III) }\end{array}$ & $\begin{array}{l}\text { Suelo muy denso y } \\
\text { roca blanda (tipo C) }\end{array}$ \\
\hline Vs10L & 342.65 & $\begin{array}{l}\text { Suelo moderadamente } \\
\text { blando (tipo III) }\end{array}$ & Suelo firme (tipo D) \\
\hline Vs11L & 633.01 & Suelo firme (tipo II) & $\begin{array}{l}\text { Suelo muy denso y } \\
\text { roca blanda (tipo C) }\end{array}$ \\
\hline Vs13L & 347.28 & $\begin{array}{l}\text { Suelo moderadamente } \\
\text { blando (tipo III) }\end{array}$ & Suelo firme (tipo D) \\
\hline
\end{tabular}

(MTI, 2007;FEMA, 2003)

Para obtener los valores de Vs30 de la tabla 2, se aplicó la ecuación 1 para cada perfil estudiado, la tercer y cuarta columna muestran la clasificación para cada uno de los sitios explorados según el RNC-07 y la norma NEHRP (FEMA, 2003), respectivamente. 
Figura 4. Modelo unidimensional de ondas de cortante en los sitios seleccionados estimados con el método MASW.
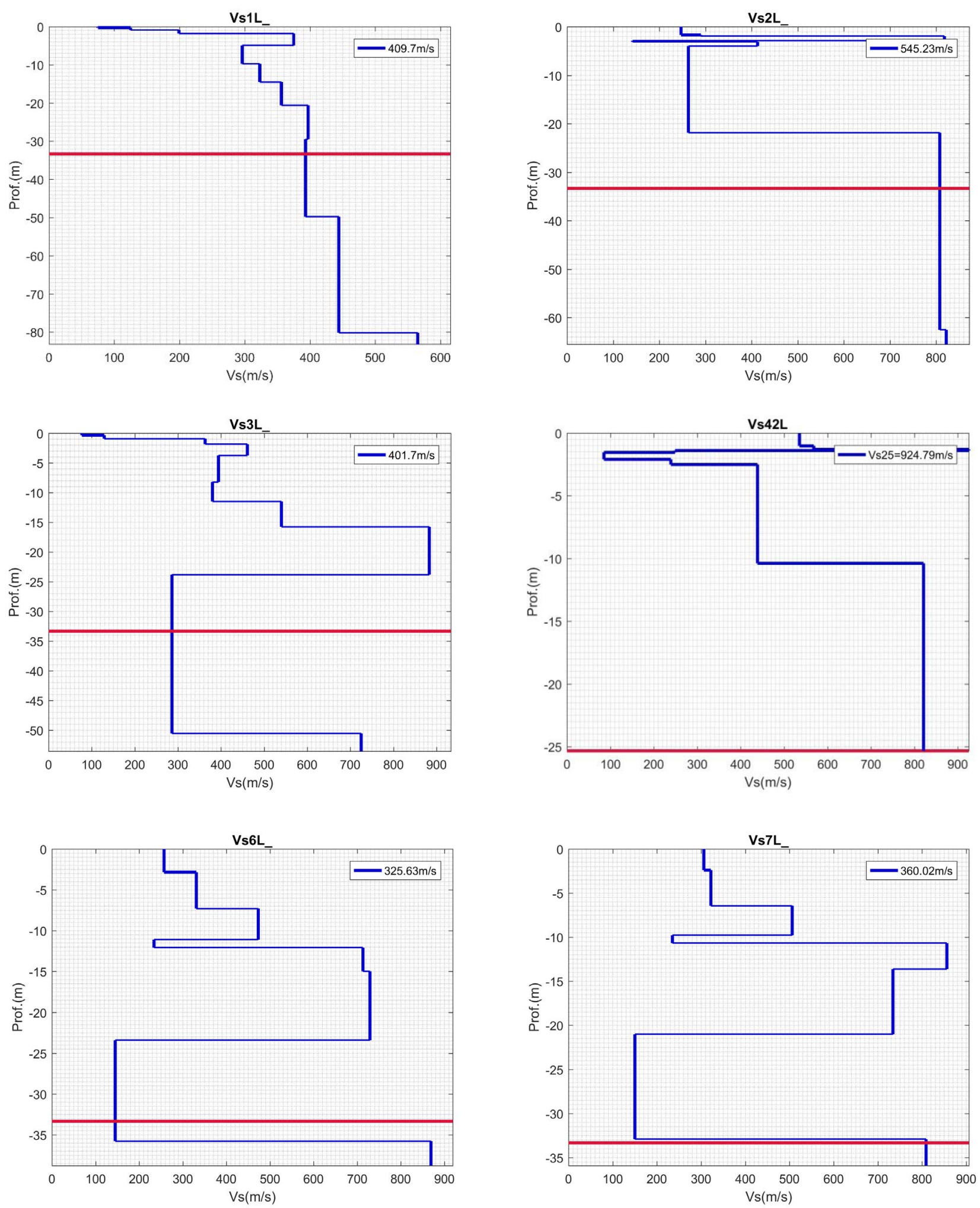
Revista Científica de FAREM-Estelí. Año 10 | Núm. 40 | Octubre-diciembre, 2021 | Pág. 96-117
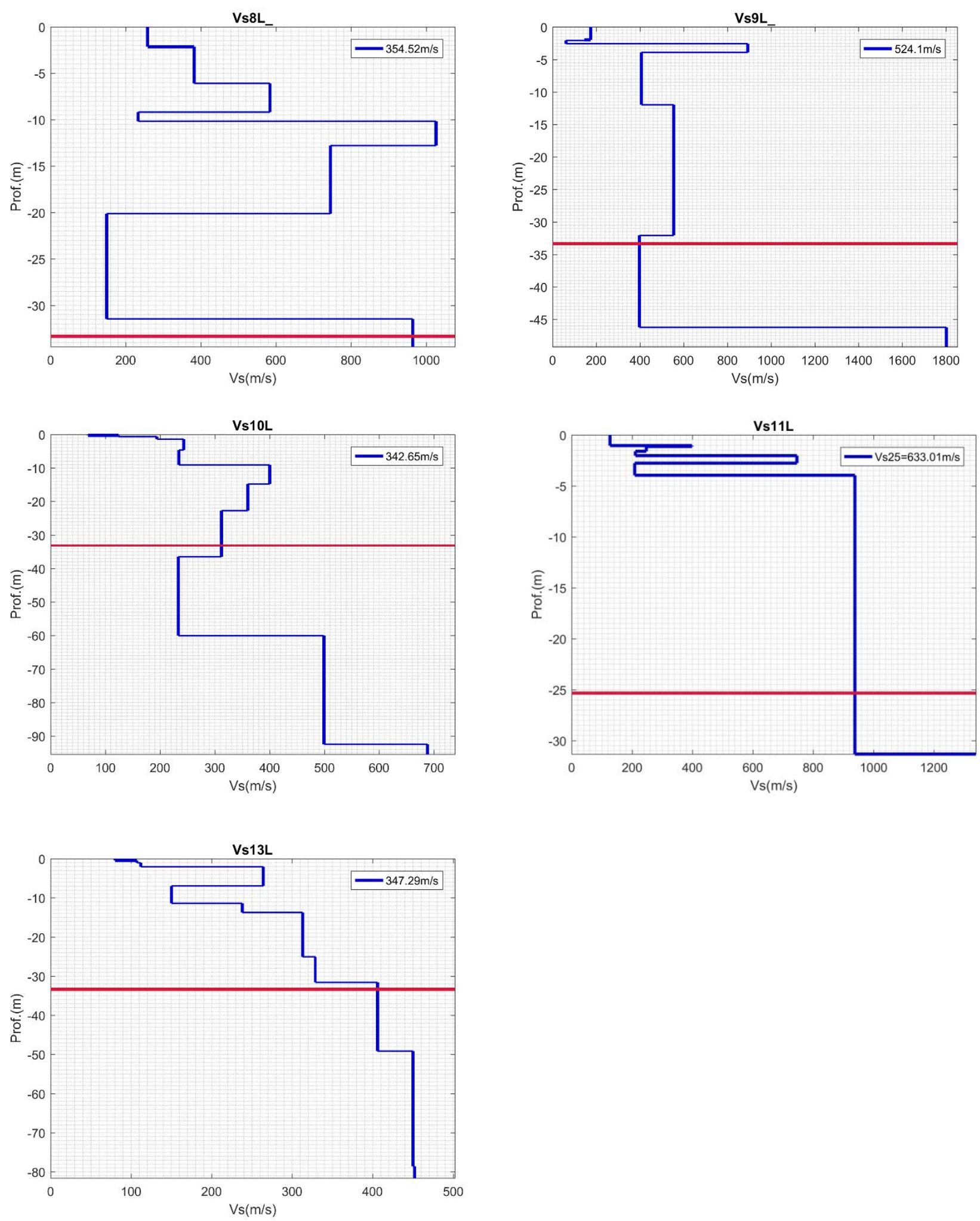


\section{Figura 5. Mapa de microzonificación sísmica del área de estudio basado en Vs30.}

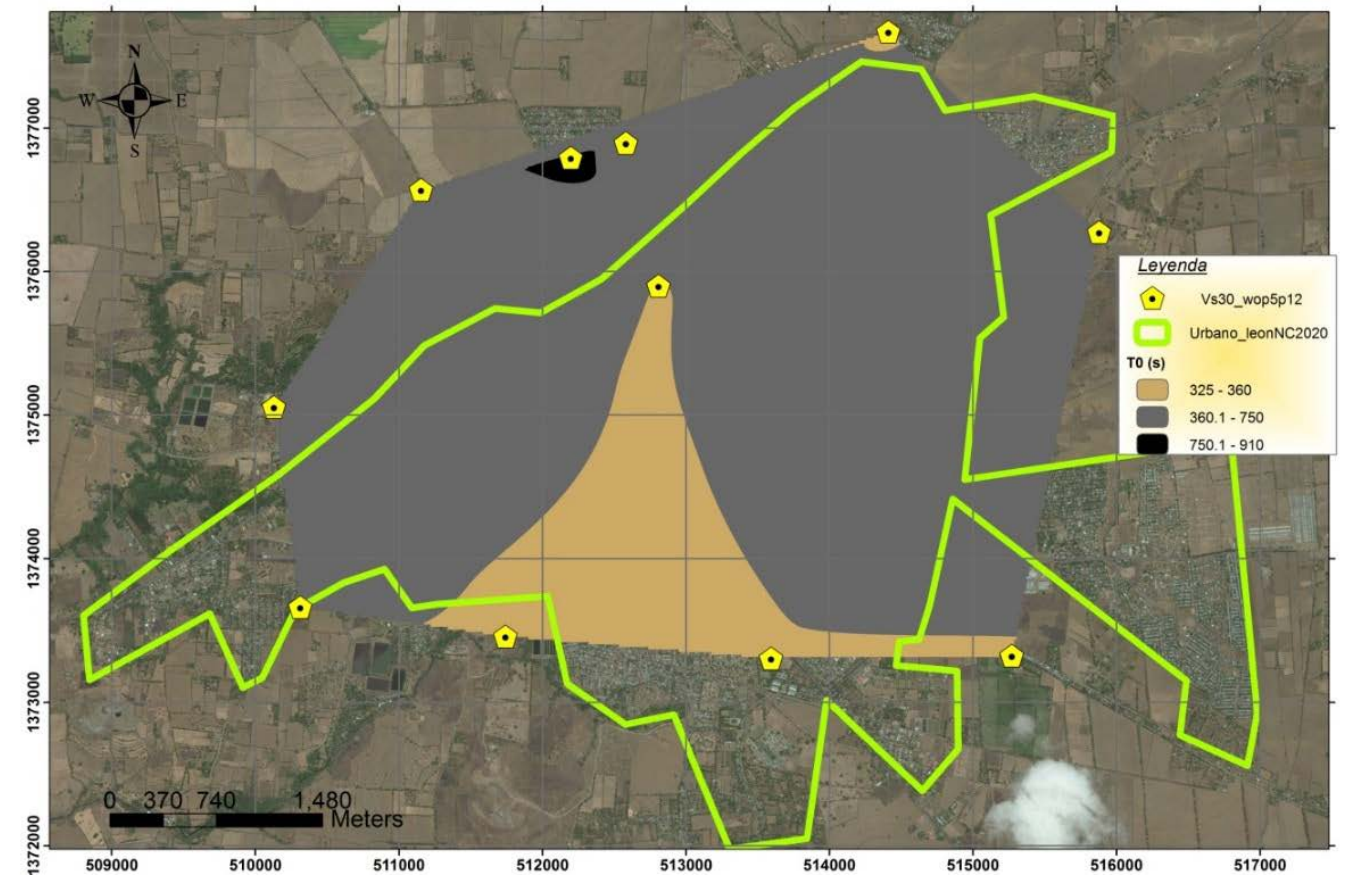

(Imagen satelital ESRI, 2021)

Teniendo como base la clasificación sísmica, se puede observar que el área ha quedado dividida en dos microzonas basándonos en el parámetro Vs30 y en el reglamento nacional de la construcción (MTI, 2007) como sigue:

Microzona 1: Presenta el parámetro de Vs30 con valores entre 325 a 360 $\mathrm{m} / \mathrm{s}$. Este suelo de acuerdo al reglamento nicaragüense de construcción puede clasificarse como suelo moderadamente blando o tipo III.

Microzona 2: Presenta el parámetro de Vs30 con valores entre 360.1 a 750 $\mathrm{m} / \mathrm{s}$. Este suelo de acuerdo al reglamento nicaragüense de construcción puede clasificarse como suelo firme o tipo II.

Únicamente un sitio presento un rango de Vs30 superior a la microzona 2 por lo cual no se agrupo en ninguna microzona. El material presente en este sitio puede clasificarse de acuerdo al reglamento nicaragüense de construcción como afloramiento rocoso o tipo $\mathrm{I}$.

\section{Espectro de diseño}

El espectro de diseño fue obtenido a partir de la ecuación 2, sugerida en el RNC-07 (MTI, 2007) (Figura 5) y fue comparado con los espectros de respuestas de terremotos seleccionados (ver tabla 1) obtenidos mediante el método de interpolación de la excitación y utilizando los datos de las propiedades dinámicas de los suelos insitu.

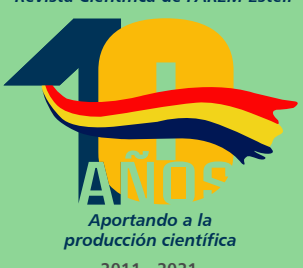


Figura 6. Comparación de espectros de respuesta con los espectros de diseño calculados para cada sitio de análisis.
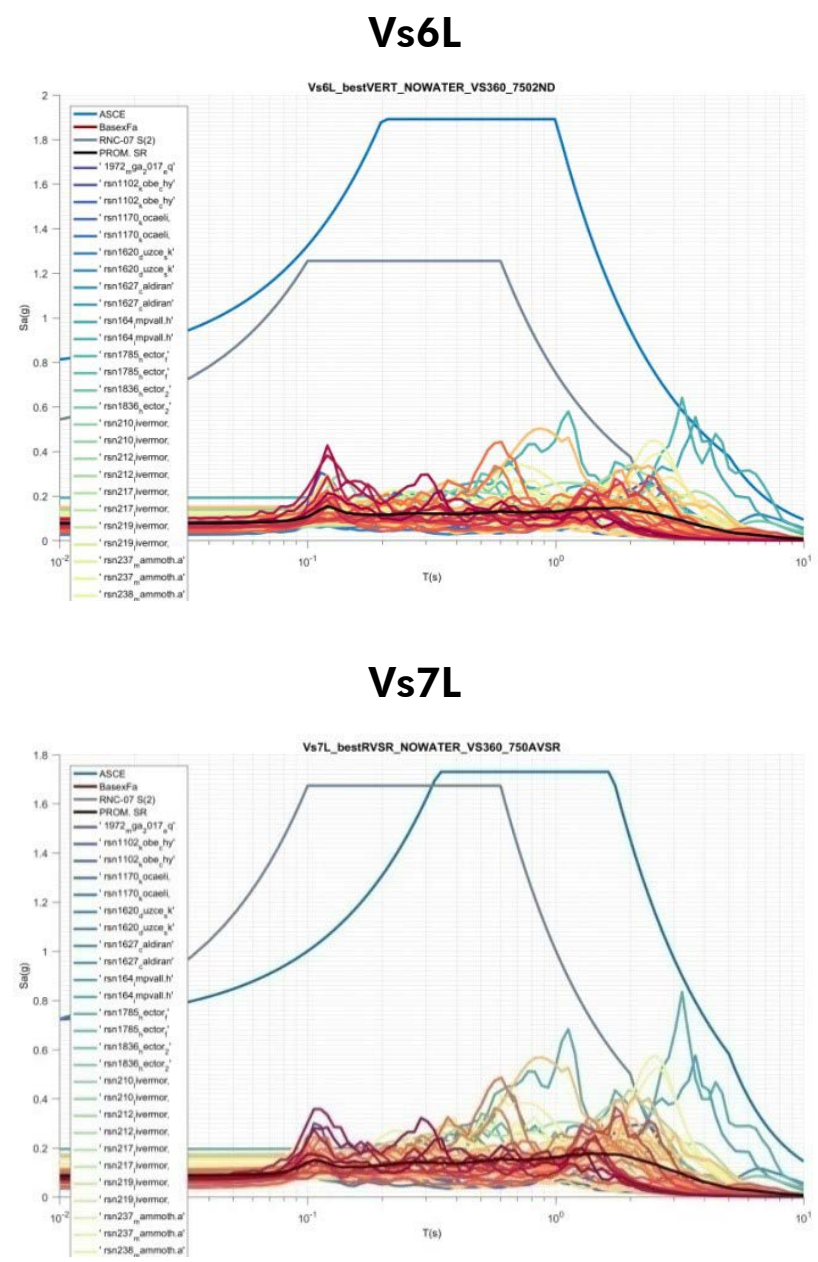

Además, se calcularon espectros de diseños adicionales utilizando las normas de la American Society of Civil Engineers en su edición de 2016 (Figura 5). Al comparar los espectros de respuesta en los sitios donde se calculó respuesta de sitio se observó claramente que había similitud en la respuesta de los sitios estudiados tanto en contenidos de frecuencia como en amplitudes. Por lo anterior, se sugiere en esta investigación utilizar un único espectro de diseño tomando como referencia las amplitudes, en este caso proponemos utilizar el espectro de diseño obtenido usando las normativas del ASCE para el sitio "Vs7L" (Figura 6). 


\section{Figura 7. Espectro de diseño sugerido para el area estudiada.}

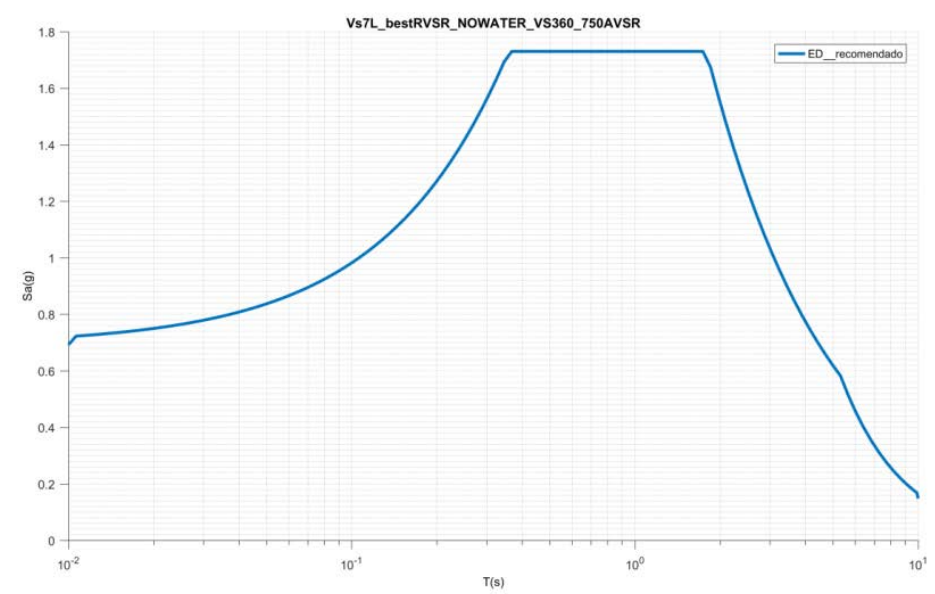

Adicionalmente, se encontró que los registros de terremotos con contenido de alta frecuencias son cubiertos adecuadamente respecto a la ubicación de la mesa del espectro de diseño del RNC-07 (MTI, 2007). Sin embargo, en el caso de Terremotos con frecuencias bajas las amplitudes sobrepasan el espectro calculado usando la formulación del RNC-07 (MTI, 2007), por lo cual se sugiere que, para edificaciones más altas, e.g. de once pisos en adelante, se realicen estudios adicionales.

Otro resultado muy importante de esta investigación fue que se encontró basados en nuestros análisis, que carece de sentido basar los cálculos de espectros de diseño en una aceleración pivote como aún se hace en el RNC07 (MTI, 2007). En su lugar, recomendamos basar los cálculos del espectro de diseño en una aceleración espectral con las mayores amplificaciones, así como en factores de amplificación correspondientes a esas aceleraciones espectrales.

Es importante mencionar que el espectro sugerido en esta investigación para la ciudad de León, es válido únicamente para terremotos locales, es decir que es necesario realizar un estudio similar para terremotos lejanos, y para depósitos de suelos más profundos, dicho estudio será desarrollado próximamente por los autores. En general puede decirse que, dado el contenido de frecuencias de los terremotos utilizados en el análisis, el espectro de diseño propuesto en este estudio es aplicable a estructuras de 1 a 10 pisos, siempre que se ubiquen en la zona estudiada. 


\section{CONCLUSIONES}

A partir de los análisis realizados en esta investigación, los hallazgos más importantes se pueden resumir en los siguientes enunciados:

1. Las funciones de transferencia empíricas (FTE) mostraron que los suelos del sitio vibran con tres modos, los cuales varían su periodo de vibración entre los valores To $1=0.049$ a $0.160 \mathrm{~s}$, To $2=0.161$ a $0.330 \mathrm{~s}$ y To3 $=0.331$ a $0.969 \mathrm{~s}$, estas constituyen las tres microzonas definidas mediante periodos fundamentales de vibración.

2. Basado en los resultados de Vs 30 se definieron las microzonas de acuerdo a microzona 1 presenta el parámetro de Vs30 con valores entre 325 a $360 \mathrm{~m} / \mathrm{s}$ y la microzona 2: Presenta el parámetro de Vs30 con valores entre 360.1 a $750 \mathrm{~m} / \mathrm{s}$.

3. La clasificación sísmica de los suelos del área de estudio se definió basado en Vs30 y en el RNC-07 según los cuales los suelos de la zona de estudio pueden clasificarse como suelos firmes o suelo moderadamente blando o como tipo II y III, respectivamente. Al comparar con las normas NEHRP los suelos del área pueden clasificarse como suelo muy denso y roca blanda o como suelo firme, o usando la nomenclatura tipo $C$ y $D$, respectivamente.

4. Se encontró a través del análisis de los espectros de diseño, que carece de sentido basar los cálculos de espectros de diseño en una aceleración pivote como aún se hace en el RNC-07. En su lugar, recomendamos basar los cálculos del espectro de diseño en una aceleración espectral con las mayores amplificaciones, así como en factores de amplificación correspondientes a esas aceleraciones espectrales.

5. Es importante mencionar que este espectro es válido para terremotos locales, es decir que es necesario realizar un estudio similar para terremotos lejanos, y para depósitos de suelos más profundos, dicho estudio será desarrollado próximamente por los autores de este estudio. En general puede decirse que, dado el contenido de frecuencias de los terremotos utilizados en el análisis, el espectro de diseño sugerido en este estudio para la ciudad de León es aplicable a estructuras de 1 a 10 pisos, siempre que se ubiquen en la zona estudiada.

\section{AGRADECIMIENTOS}

El autor desea expresar su agradecimiento especial a la Universidad Nacional Autónoma de Nicaragua (UNAN-Managua) que a través de los fondos para proyectos de investigación (FPI) apoyo económicamente la realización de este estudio. 
También agradece al Instituto de Geología y Geofísica que apoyo el proyecto en gestiones de logística y administrativas.

Finalmente, quiero agradecer a mi estudiante Christian Aguilar por apoyarme en la etapa de adquisición de datos.

\section{REFERENCIAS}

Alfaro, A., Navarro, M., Sánchez, J., \& Pujades, L. (1999). Microzonificación Sísmica de Barcelona utilizando el método de Nakamura. Congreso Nacional de Ingeniería Sísmica, 273-278. http://www.ciees.org/doc_ estudios/1999_alfaro navarro_sanchez_pujades.pdf

Ancheta, T. D., Darragh, R. B., Stewart, J. P., Seyhan, E., Silva, W. J., Chiou, B. S.-J., Wooddell, K. E., Graves, R. W., Kottke, A. R., Boore, D. M., Kishida, T., \& Donahue, J. L. (2014). NGA-West2 Database. Earthquake Spectra, 30(3), 989-1005. https://doi.org/10.1193/070913EQS197M

Arslan, H., \& Siyahi, B. (2006). A comparative study on linear and nonlinear site response analysis. Environmental Geology, 50(8), 1193-1200. https:// doi.org/10.1007/s00254-006-0291-4

Baker, J. W., \& Lee, C. (2018). An Improved Algorithm for Selecting Ground Motions to Match a Conditional Spectrum. Journal of Earthquake Engineering, 22(4), 708-723. https://doi.org/10.1080/13632469.2016 .1264334

Bonnefoy-Claudet, S., Cornou, C., Bard, P. Y., Cotton, F., Moczo, P., Kristek, J., \& Fäh, D. (2006). H/V ratio: A tool for site effects evaluation. Results from 1-D noise simulations. Geophysical Journal International, 167(2), 827-837. https://doi.org/10.1111/j.1365-246X.2006.03154.x

Castrillo-Osorio, N. (2021). Avances en la microzonificación sísmica de la región del Pacifico de Nicaragua. In SOVG. (Ed.), XVI Congreso Venezolano de Geofísica.

Castrillo E. N. Yokoi T., \& Ulriksen P, E. K. (2014). Local site effect characterization in the old downtown area of Managua city, Nicaragua. 5th Asian Conference on Earthq. Eng.

FEMA. (2003). Nehrp Recommended Provisions for Seismic Regulations for New Buildings and Other Structures (Fema 450). In Part 1 (Issue Fema 450).

Hernandez, O., \& Lermo, J. (2009). Reevaluación del efecto de sitio y propuesta de clasificación de terreno con fines de diseño sísmico para Managua, Nicaragua. In IGG-CIGEO: Vol. Máster. UNAN-Managua.

Hodgson, G. (2000). Geología Regional de Nicaragua. In Introducción al Léxico Estratigráfico de Nicaragua.

INETER. (2000). Microzonificación sísmica de Managua.

INETER. (2004). Mitigación de Geo-riesgos. 
INIDE. (2020). Anuario estadístico.

Kagawa, T. (1996). Estimation of velocity structures beneath Mexico City using microtremor array data. In P. Oxford (Ed.), 11th World Conference on Earthquake Engineering.

Kanai Tanaka, T., K. (1961). On microtremor VIII. Bulletin Earthquake Research Institute.

Lacayo, L. (2016). Evaluación del efecto de sitio en el area urbana de la ciudad de León. In Instituto de Geología y Geofísica de la UNAN-Managua. UNAN-Managua.

Martínez, D. (2011). Effect of surface geology (considering nonlinearity of subsoil) on ground motion in the urban area of managua, nicaragua. In IISEE-BRI: Vol. Máster. GRIPS.

MOVIMONDO. (2004). Amenazas geológicas y vulnerabilidad de la ciudad de León.

MTI. (2007). Reglamento nacional de la construcción, RNC-07. (D. general de N. de construcción y desarrollo urbano. (ed.)).

Nakamura, Y. (1989). Method for dynamic characteristics estimation of subsurface using microtremor on the ground surface. Quarterly Report of RTRI (Railway Technical Research Institute) (Japan), 30(1), 25-33.

Nogoshi M., Igarashi, T. (1971). On the amplitude characteristics of microtremors (part 2). Journal of Seismological Society of Japan.

Obando, E., Ryden, N., Park, C., Ulriksen, P. (2011). A depth mobile for measuring transfer functions. Soil Dynamics and Earthquake Engineering.

Park, C. B., Miller, R. D., \& Xia, J. (1999). Multichannel analysis of surface waves. Geophysics, 64(3), 800-808. https://doi.org/10.1 190/1.1444590

Parrales, R., \& Ulriksen, P. (2006). Dynamic properties of the soils in the area of Managua, Nicaragua. In Geology department: Vol. Licentiate. Lund.

Romero, C. (2005). Estudio de efecto de sitio en el area urbana de las ciudades de Masaya y Catarina. In IGG-CIGEO: Vol. Máster. UNAN-Managua.

Schnabel Lysmer, J., \& Seed, H. B., P. B., \& California, U. of. (1972). A computer Program for Earthquake Response Analysis of Horizontally Layered Sites.

Shah Mortgat, C., Kiremiijian, A., \& Zsutty, T., H., \& university, S. (1975). A study of seismic risk for Nicaragua, Part I.

Tuladhar, R., Yamazaki, F., Warnitchai, P., \& Saita, J. (2004). Seismic microzonation of the greater Bangkok area using microtremor observations. Earthquake Engineering and Structural Dynamics, 33(2), 211-225. https://doi.org/10.1002/eqe.345

Wathelet, M., Chatelain, J. L., Cornou, C., Giulio, G. Di, Guillier, B., Ohrnberger, M., \& Savvaidis, A. (2020). Geopsy: A user-friendly opensource tool set for ambient vibration processing. Seismological Research Letters, 91 (3), 1878-1889. https://doi.org/10.1785/0220190360

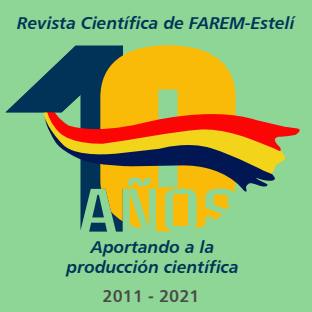

